\title{
IMMUNE NAVIGATION CONTROL FOR STIGMERGY BASED FORAGING BEHAVIOUR OF AUTONOMOUS MOBILE ROBOTS
}

\author{
Diana Tsankova*, Velichka Georgieva*, Frantisek Zezulka**, Zdenek Bradac** \\ * Control Systems Dept., Technical University - Plovdiv, 25 Ts. Dyustabanov Str, \\ 4000 Plovdiv,Bulgaria,email: tsankova@tu-plovdiv.bg,villy07@yahoo.com, \\ ** Department of Control and Instrumentation, Brno University of Technology, \\ Kolejni 4, 61200 Brno, Czech Republic, e-mail: zezulka@feec.vutbr.cz, \\ bradac@feec.vutbr.cz
}

\begin{abstract}
The paper presents a series of experiments in a simulated environment where two autonomous mobile robots gather randomly distributed objects and cluster them into one pile. The coordination of the robots' movements is achieved through stigmergy (an indirect form of communication through the environment). In order to avoid the drawback of the random moves, necessary for stigmergy based foraging behaviour, the perceptive capabilities of the robots are enhanced by detectors for concentration of objects. An artificial immune network carries out the collision free goal following behaviour. Simulations confirm the improved performance of the foraging behaviour under the proposed immune navigation control. Copyright $@ 2005$ IFAC
\end{abstract}

Keywords: foraging behaviour, stigmergy, immune network, autonomous mobile robot.

\section{INTRODUCTION}

The concept of stigmergy has been introduced by the French entomologist Pierre-Paul Grassé (1959) in the 1950 s during his studies of nest building in termites. Stigmergy is derived from the roots "stigma" (goad) and "ergon" (work), thus giving the sense of "incitement to work by the products of work" (Beckers, et al., 1994). The stigmergic principle appears to organize corpse-gathering behaviour in ant colonies. The emergence of corpse clusters has been studied by Deneubourg, et al. (1990). Stigmergy is an indirect means of communication between multiple mobile agents involving modifications made to the environment. The environment itself acts as an external memory in the higher level context of the system as a whole but the existence of only one local stimulus is needed to drive agent behaviour. The agent is programmed so that it obeys a simple set of rules and recognizes local information to perform a small task.
The stigmergic principles can be successfully applied to robotics, especially for an object foraging task. Random movements are needed in order to ensure an exploration of all the places of the arena within a reasonable period of time. In order to avoid the drawback of the random moves (loss of time wandering around in an area without objects), the perceptive capabilities of the robots have been enhanced by giving them detectors for concentration of objects (Tsankova and Georgieva, 2004). The type and characteristics of these simulated detectors influence the speed of the foraging process. Because of detectors' uncertainty (count objects, disposed on a determinate sector, but do not discriminate clusters among them), in some cases the robot can fall into a trap picking up objects from one place and dropping them approximately on the same place. A little "exploration" of the environment could be of benefit for speeding up the clustering. 
In robotics, artificial immune systems are proposed by Ishiguro, et al. (1995a,b) to solve, mostly, collision free goal following navigation tasks. In such immune system competence modules (antibodies) form a network with stimulation and suppression connections. The immune network can be viewed as a mechanism for action selection on a system level. The paper proposes an approach using the advantages of the artificial immune system and the enhanced sensing of the concentration of objects for speeding up the stigmergy based foraging behaviour of mobile robots. Simulations in MATLAB environment, involving one robot working alone, and two robots working simultaneously, confirm the improved performance of the proposed robot control.

\section{TASK FORMULATION AND A SET OF RULES}

A system of two robots for gathering a scattered set of objects (pucks) into a single cluster (like the corpse-gathering behaviour of ants) is developed. To achieve this task by stigmergy, a simulated robot can be designed to move objects that are more likely to be left in locations where other objects have previously been left. For this purpose the robot is equipped with a simple threshold mechanism - a gripper, able to sense one puck. An additional detector for puck concentration is used to determine the directions (in respect to the robot) with maximum and minimum (non-zero) concentrations of pucks (Tsankova and Georgieva, 2004b). This information is needed to prevent the random walks and to speed up the clustering process. The idea is the robots to pick up pucks from places with small concentration and to drop them on places with high concentration of pucks. Two types of stigmergy based controls with enhanced sensing of object concentration are discussed: the first one includes simple rules with fixed priorities, and the second one uses an immune network for a collision free goal following behaviour. The plan is to evaluate the performance of the robots equipped with the above mechanisms and controls in simulations.

Consider a mobile robot with two driving wheels, mounted on the same axis, and a front free wheel (Fig.1a). Radii of the robot and the puck are $R=0.036 \mathrm{~m}$ and $R_{\text {puck }}=0.015 \mathrm{~m}$, respectively.

Each robot carries a U-shaped gripper with which it can take pucks. The robots are run in a square area $0.9 \mathrm{~m} \times 0.9 \mathrm{~m}$. Before starting each run, 49 pucks are placed in the form of a regular grid in the arena, as shown in Fig.5a. The robots are equipped with simulated obstacle detectors (five IR sensors) and a simulated microswitch, which is activated by the gripper when one puck is picked up. Obstacle detectors are installed in five directions shown in Fig.1b. They can detect the existence of obstacle in their directions (sectors $S_{i}, i=1,2, \ldots, 5$ ), and the detectable range of sensors is assumed to be equal to the diameter of the robot. The detectors for puck concentration are also collocated at the same position as the obstacle detectors (Fig.1b). The simulated detector for concentration of pucks can enumerate the pucks (but does not discriminate clusters), which are disposed in the corresponding sector $S_{i}$ with range covering the entire arena. The readings of the detectors for puck concentration are normalized as

$$
D_{i}=N_{i}^{\text {puck }} / n^{\text {puck }}, \quad i=1,2, \ldots, 5,
$$

where $D_{i}$ is the reading of the detector for the concentration of pucks, corresponding to the sector $S_{i} ; N_{i}^{\text {puck }}$ - the number of pucks, located in the sector $S_{i} ; n^{\text {puck }}-$ all 49 pucks.

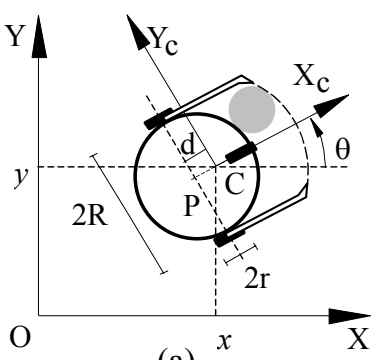

(a)

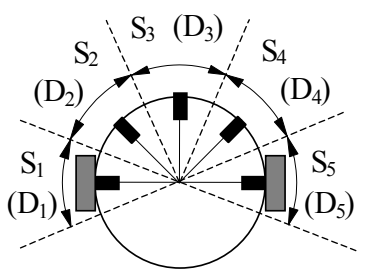

(b)
Fig. 1. Autonomous mobile robot.

The following set of rules describes the basic behaviour of the robots, taking into account the puck density:

Rule 1. If (there is not a puck in the gripper) \& (a puck ahead) then take one puck in the gripper.

Rule 2. If (there is one puck in the gripper) \& (a puck ahead) then drop a puck and go backward for one second.

Rule 3. If (there is not a puck in the gripper) \& (there are no pucks ahead) then follow the direction, corresponding to the minimum (non-zero) reading of the detectors for puck concentration.

Rule 4. If (there is one puck in the gripper) \& (there are no pucks ahead) then follow the direction, corresponding to the maximum reading of the detectors for concentration of pucks.

Rule 5. If there is an obstacle (wall or other robot) ahead then avoid the obstacle (turn on the obstacle avoidance behaviour).

When no obstacle detector is activated, the robot executes a goal following behaviour with an artificial goal corresponding to the place with maximum or minimum concentration of pucks, depending on whether or not there is a puck in the gripper, respectively. The puck concentration detectors determine the direction of the artificial goal. If all pucks are disposed behind the robot, the low-level control makes the robot turn until a puck concentration detector becomes active. The goal following behaviour continues until an obstacle is detected or the microswitch is activated (pucks are not detected as obstacles). On detecting an obstacle, the robot executes the obstacle avoidance behaviour. 
On the spot it turns from the obstacle away at a random angle until detectors no longer find out the obstacle, and then the robot goes forward (Beckers, et al., 1994). If the robot carries a puck, when it encounters the obstacle, the gripper will retain the puck during the turn. When the gripper tries to pick up two or more pucks the microswitch triggers the puck-dropping behaviour. This behaviour consists of releasing the puck from the gripper and going backwards for a second. The obstacle avoidance behaviour has priority over the puck-dropping one.

In conformity with the proposed stigmergy based control using immune navigator, the above set of rules (from Rule 1 to Rule 5) is modified as the first two rules remain unchanged, and the rest are substituted by the following Rule $3 a$ :

Rule $3 a$. If (there are no pucks ahead) OR (there is an obstacle ahead) then turn on the collision free goal following behaviour, realized by an artificial immune network.

The immune network implements a goal following behaviour, an obstacle avoidance one and arbitrates them. If there is one puck in the gripper the direction of the goal is equal to the direction corresponding to the sector with maximum number of pucks, and if there is no puck in the gripper - the direction with minimum puck concentration.

At the beginning of each of a number of experiments, the robots start from a random initial position and orientation. Every minute of runtime, the robots are stopped, the sizes and positions of clusters of pucks are recorded, and the robots are restarted. The experiment continues until all 49 pucks are in a single cluster. A cluster is defined as a group of pucks separated by no more than one puck diameter (Beckers, et al., 1994). For the simplicity of simulations the following assumptions in the design of the gripper, the microswitch and the pucks are used (Tsankova and Georgieva, 2004a):

- A puck will be scooped, only when it fits neatly inside the semicircular part of the gripper.

- If part of a puck is outside of the gripper, the puck will not be scooped, it will not be pushed aside, and the robot will pass across it.

- When the microswitch is activated the puck may be dropped either on an empty area or on other pucks.

- The pile may grow in height.

\section{IMMUNE NETWORK}

B-lymphocytes (basic components of the immune system) have distinct chemical structure and produce "Y" shaped antibodies. The antibody recognizes an antigen like a key and lock relationship. The structure of the antigen and the antibody is shown in Fig.2, where the portion of the antigen recognized by the antibody is called epitope, and the portion of the antibody that recognizes the corresponding antigen determinant is called paratope. Antibodies also have antigenic characteristic called idiotope and key and lock relationship also exists between different species of antibodies. Based on this fact antibodies communicate to each other, and the stimulation and suppression chains among them form a large-scaled network, i.e. the immune system provides a parallel distributed processing architecture.

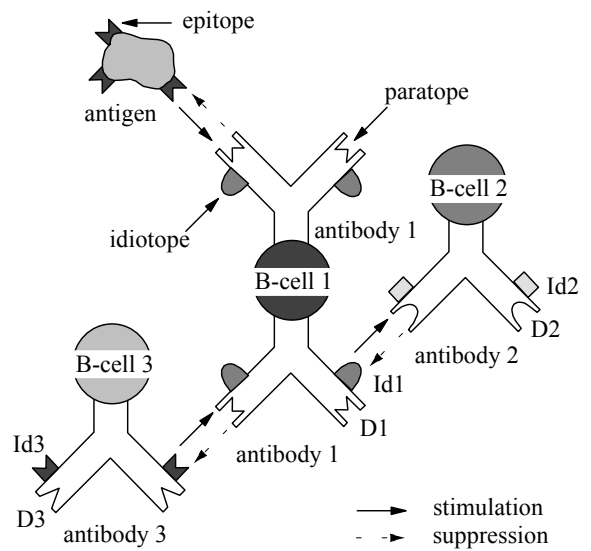

Fig.2. Structure of immune network (Ishiguro, et al., 1995a).

Consider a goal following and obstacle avoidance navigation task. At the current situation, for example, distance and direction to the detected obstacle or to the goal works as an antigen, the competence module (simple behaviour/action) can be considered as an antibody, and the interaction between modules is presented as stimulation/suppression between antibodies. The concentration $a_{i}(t)$ of $i$-th antibody is calculated as (Ishiguro, et al., 1995a):

$$
\frac{d a_{i}(t)}{d t}=\left(\frac{\sum_{j=1}^{N} m_{j, i} a_{j}(t)}{N}-\frac{\sum_{k=1}^{N} m_{i, k} a_{k}(t)}{N}+m_{i}-k_{i}\right) a_{i}(t),(2)
$$

where $N$ is the number of antibodies, $m_{j, i}$ and $m_{i}$ denote affinities between the antibody $j$ and the antibody $i$, and the antibody $i$ and the detected antigen, respectively. The first and the second terms on the right hand side denote the stimulation and suppression from other antibodies, respectively. The third term represents the stimulation from the antigen, and the fourth term $k_{i}$ - the natural death. The affinity coefficients $m_{j, i}$ and $m_{i}$ are calculated by (Ishiguro, et al., 1995a):

$$
\begin{aligned}
m_{j, i} & =\alpha \sum_{k}^{L} I_{j}(k) \oplus \overline{P_{i}(k)}, \\
m_{i} & =\beta \sum_{k}^{L} E(k) \oplus \overline{P_{i}(k)},
\end{aligned}
$$

where $\alpha$ and $\beta$ are positive constants, $\oplus$ represents exclusive-or operator, $L$ is the length of the 
paratope, the idiotope and the epitope written as binary strings. $I_{j}(k), P_{i}(k)$ and $E(k)$ represent the $k$-th binary value in the idiotope string of antibody $j$, the paratope string of antibody $i$, and the epitope string, respectively. If the concentration of the antibody exceeds an a priori signed threshold, the antibody is selected and its corresponding behaviour becomes acting to the world.

The obstacle detectors give binary information $1 / 0$, about existence or absence of obstacles in their range, respectively. On the base of the normalized readings of the puck concentration detectors $D_{i}, i=1,2, \ldots, 5$ a simulated goal detector can recognize the direction of destination (maximum/minimum puck heaping) at any position of obstacle detectors. In the case, in which there is a puck in the gripper, the simulated goal detector responds with 1 to the direction of $D_{\max }=\max \left(D_{i}\right)$ and with 0 to the rest. When the robot does not carry a puck, it responds with 1 to the direction of $D_{\min }=\min \left(D_{i}\right)$ and 0 to the rest. Therefore, the robot's simulated detectors discover two types of antigens (obstacle-oriented antigens and goal-oriented ones), and each antigen has five-bit epitope. The antigens inspire the same two types of antibodies. The antibody's paratope (Fig.3) corresponds to the desirable condition (the precondition, which has to be fulfilled before the antibody can be activated), and its idiotope - to the disallowed antibodies (this antibodies which are impossible or undesirable, when the condition of the paratope and its corresponding action are implemented). For mobile robot navigation a simple immune network with 12 a priori prepared antibodies is used (Fig.4). The first six antibodies are stimulated from obstacle-oriented antigens, and the other six from goal-oriented ones. The actions are: move forward (Front), turn right (RS, RM), turn left (LS, LM), move backward (TurnBack). In Fig.4 to facilitate the explanation of the network, goaloriented paratopes are not presented as binary strings, but in calculations they are replaced with such ones. For example, $\mathrm{G} \in \mathrm{S}_{1}$ is replaced with 10000 and denotes that the goal $(\mathrm{G})$ appears in the sector $\mathrm{S}_{1}$ of the goal detector, and $G \in$ none - with 000000 and shows that the goal is not discovered in the five sectors of the goal sensor, i.e. it is behind the robot. The symbol \# denotes that the condition can be taken as either 0 or 1 , i.e. it can be regarded as not so important information. Therefore, in (3) and (4), when $P_{i}(k)=\#$ or $I_{j}(k)=\#$ it determinates that $I_{j}(k) \oplus \overline{P_{i}(k)}=E(k) \oplus \overline{P_{i}(k)}=0.25$. The idiotope includes disallowed antibodies for situation, in which the paratope condition is fulfilled. For example, the paratope of antibody 9 shows that the goal is discovered in front of the robot in sector $\mathrm{S}_{3}$ and the corresponding action is move forward (Front). This behaviour will be impossible, if there is an obstacle in front of the robot, i.e. the obstacle detectors react with the string \#\#1\#\#, which unites the paratopes of the antibodies 2, 3, 4, 5 and 6, and they are considered as disallowed. Readings of the puck concentration detectors form the goal-oriented antigens. For example, if the maximum puck heaping has occurred in the sector $S_{4}$, i.e. $D_{\max } \in S_{4}$, and minimum one in $S_{1}\left(D_{\min } \in S_{1}\right)$, then the epitope string will be 00010 or 10000 , corresponding to whether there is or there is not a puck in the gripper. In Fig.4 the stimulation connections from idiotopes to corresponding paratopes are shown by arrows. For each particular situation, detected by sensors, only one of all antibodies wins (in conformity with (2) (4)) and its action becomes target behaviour (direction of movement) for the mobile robot.

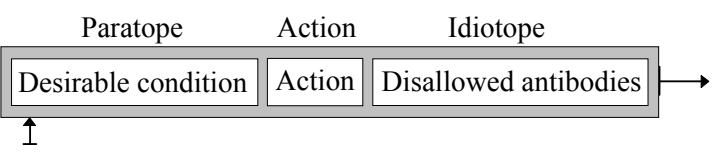

Fig. 3. Antibody (Ishiguro, et al., 1995a).

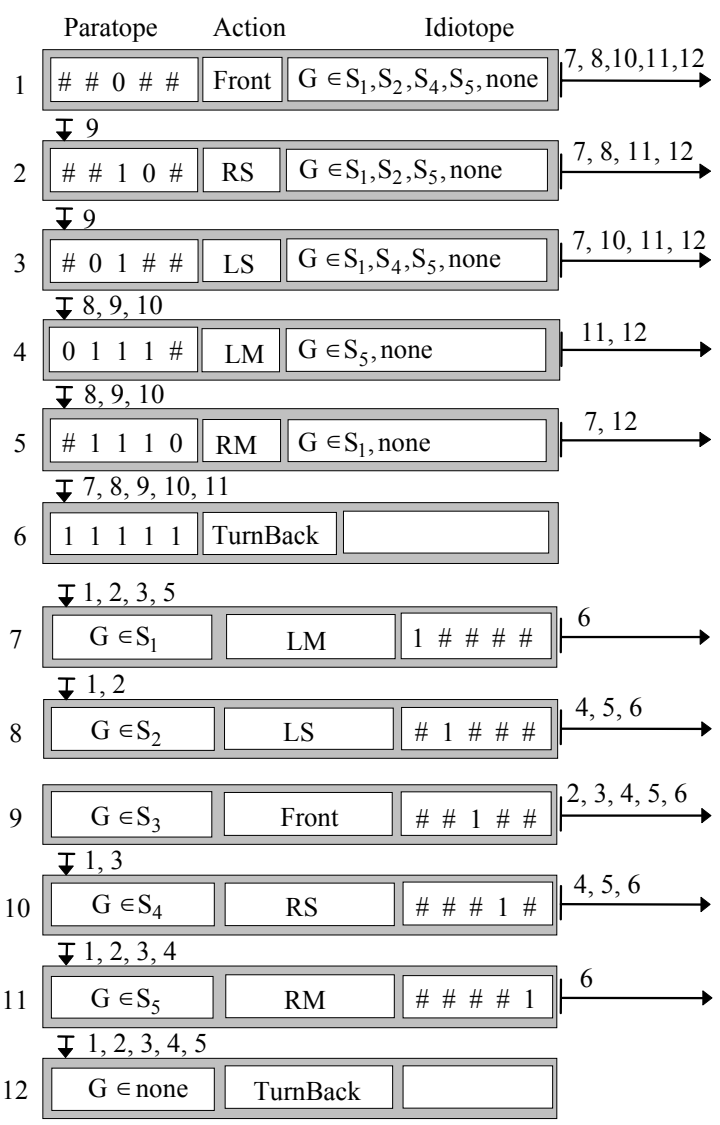

Fig. 4. Immune network for collision free goal following behaviour (Tsankova and Topalov, 1999).

\section{SIMULATION RESULTS}

To illustrate the performance of the two variants of stigmergy based robot control for a simple foraging 
task, they have been simulated in MATLAB environment and tested on several examples. The robot tracking control system under the assumption for "perfect velocity tracking" was realized as in Kanayama, et al. (1990). The foraging task included gathering pucks in a pile by one robot, working alone, and by two robots working simultaneously. Simulations of puck gathering behaviour by two robots with immune navigators are shown in Fig.5. At the start, the arena contains only single pucks (Fig.5a). The immune navigator determines the direction of movement: the most probable directions are these with maximum and minimum puck concentrations, depending on whether there is or there is not a puck in the gripper, respectively. But since the immune network makes decision at the system level, it can assign a target direction of the robot, different from minimum and maximum puck heaping directions. Thus "exploration" behaviour takes superiority over "exploitation" one. Each robot moves forward scooping one puck into the gripper and moves on following the direction given by the immune navigator. When two pucks have been gathered, the robot drops them, leaving them as a cluster, moves backwards for a second, turns at the angle, determined by the immune navigator, and goes forwards. Within a short period of time most pucks are in small clusters with less probability to be destroyed (Fig.5b). The robots remove pucks from smaller clusters by striking them at an angle with the gripper. The pucks removed in this way are added to other usually larger clusters when the robot collides with them. Some clusters grow rapidly at this stage and after some time there is a small number of relatively large clusters (Fig.5c). A single cluster is formed in the end (Fig.5d).

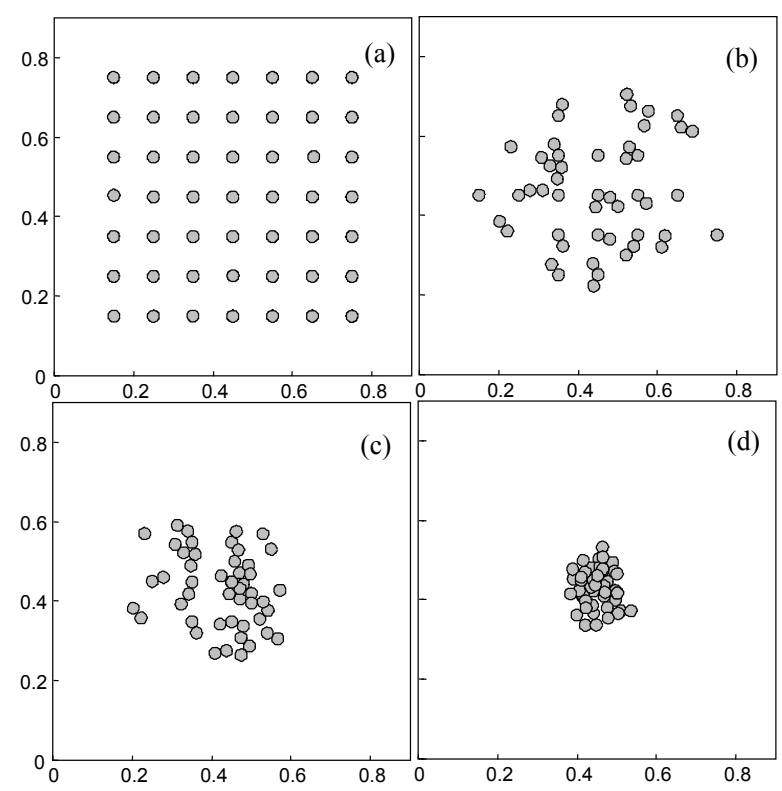

Fig. 5. The initial setup (a) and time evolution of a foraging experiment by two robots with immune navigators: (b) $6 \mathrm{~min}$, (c) $11 \mathrm{~min}$, (d) $16 \mathrm{~min}$.
The simulation results of the foraging behaviour with the proposed immune navigator, in terms of (a) number of clusters and (b) maximum cluster size from four experiments, using one robot alone or two robots working simultaneously are shown in Fig.6 and Fig.7, respectively. The average values from these experiments are shown in Fig.8. The same
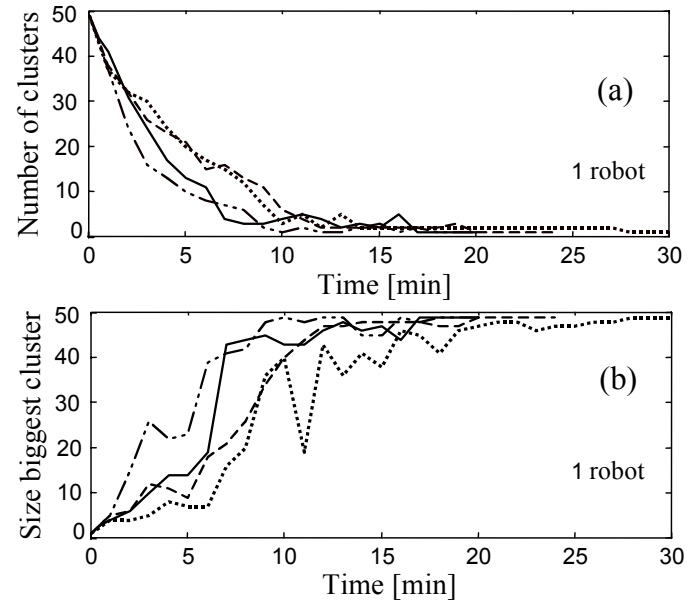

Fig 6. Simulations with the immune navigator.
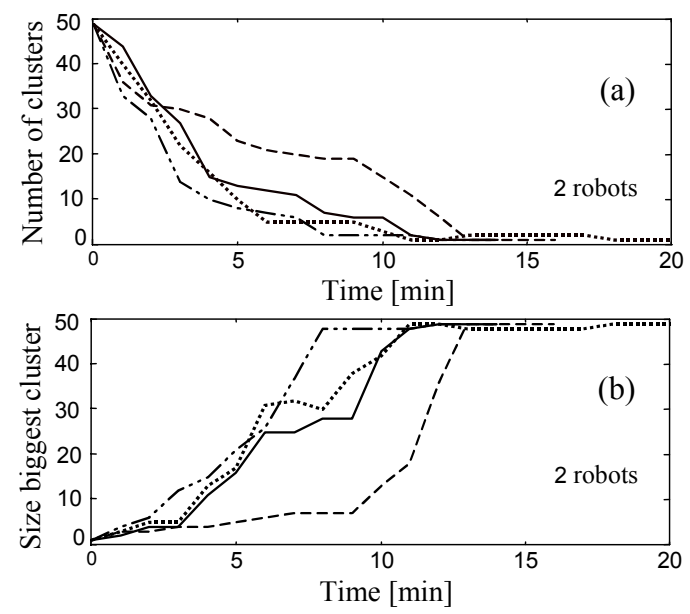

Fig. 7. Simulations with the immune navigator.
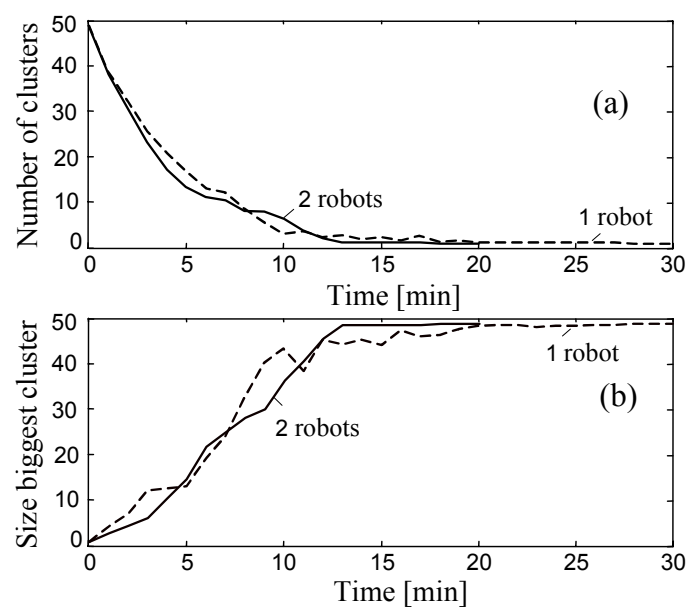

Fig. 8. The average values from experiments shown in Fig. 6 and Fig.7. 
experiments were implemented with the other control algorithm - without the immune navigator, and the average values from these four experiments are shown in Fig.9.
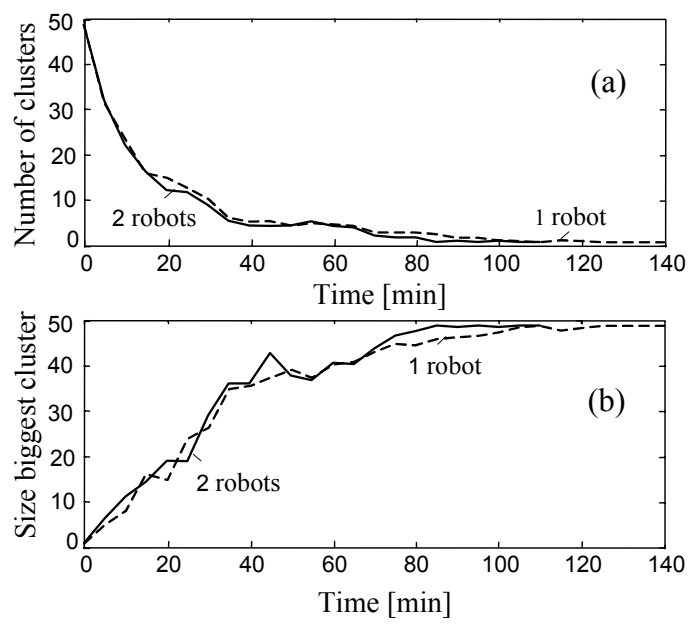

Fig. 9. The average values from experiments without the immune navigator.

Stigmergic mechanism organizes the transfer of pucks from smaller to larger clusters, even when the robots are unable to discriminate between them with their sensors (Beckers, et al., 1994). The simulated detectors for puck concentration only speed up the foraging process. They detect very roughly the directions (sensor sectors) with minimum and maximum puck concentrations, no matter whether the pucks are gathered in one or more clusters. Sometimes the robot picks up pucks from one place and drops them approximately on the same place. Roughness of the sensors for puck concentration increases the time for clustering, but nevertheless the overall time (Fig.9) remains about 4 times less than the time in the experiments, based on random walks and not taking into account puck density (Tsankova and Georgieva, 2004b). Under using immune navigator, the clustering process becomes once more faster - more than 4 times (Fig.6, Fig.7, Fig.8). Two robots, working simultaneously, usually finish the clustering faster than one robot, working alone. But in our simulations (Fig.8 and Fig.9) the benefit is insignificant, because the arena is chosen very small and the robots lost time avoiding each other quite often, and some clusters have been destroyed at this time.

\section{CONCLUSION}

The proposed stigmergy based foraging behaviour control using an immune navigator and an enhanced sensing of object concentration improves the fastness of the clustering process, which is confirmed by MATLAB simulations. When random walks, needed to ensure an exploration of the entire arena (Beckers, et al., 1994), are replaced by purposeful moves, taking into account the perceived concentration of objects, the main drawback of stigmery based approach (loss of time wandering around in an area without interest) is reduced. The detectors for puck concentration speed up the foraging process, but how to construct such real sensors is an open question. The proposed immune navigator speed up the foraging even more, because (1) it improves collision free goal following behaviour and (2) it makes decisions at the system level and can determine a goal direction different from the direction with minimum/maximum puck concentration. Thus, it compensates partially some drawbacks of the detectors for object concentration.

\section{REFERENCES}

Beckers, R., O.E. Holland and J.L. Deneubourg (1994). From Local Actions to Global Tasks: Stigmergy and Collective Robotics. In: Proc. of the Fourth Int. Workshop on the Synthesis and Simulation of Living Systems Artificial Live IV (R.Brooks and P. Maes (Eds), pp.181-189, MIT.

Deneubourg, J.L., S.Goss, N.R. Franks, A. SendovaFranks, C. Detrain and L. Chretien. (1990). The dynamics of collective sorting: robot-like ants and ant-like robots. In: Simulation of Adaptive Behaviour: from animals to animats (J-A. Meyer and S. Wilson (Eds)), pp.356-365, MIT Press.

Grassé, P.P. (1959). La reconstruction du nid et les coordinations inter-individuelles chez Bellicositermes natalensis et Cubitermes sp. La theorie de la stigmergie: Essai d'interpretation des termites constructeurs. In: Ins. Soc., Vol.6, pp.4183.

Ishiguro, A., Y.Watanabe and Y.Uchikawa (1995a). An Immunological Approach to Dynamic Behavior Control for Autonomous Mobile Robots. In: Proc. of IROS'95, Vol.1, pp.495-500.

Ishiguro, A., T.Kondo, Y.Watanabe and Y.Uchikawa (1995b). Dynamic Behavior Arbitration of Autonomous Mobile Robots Using Immune Networks. In: Proc. of ICEC'95, pp.722-727.

Kanayama, Y., Y.Kimura, F.Miyazaki and T.Noguchi (1990). A Stable Tracking Control Method for an Autonomous Mobile Robot. In: Proc. IEEE Int. Conf. on Robotics and Automation, Vol.1, pp.384-389.

Tsankova, D.D. and V.S. Georgieva (2004a). From Local Actions to Global Tasks: Simulation of Stigmergy Based Foraging Behavior. In: Proc. of the $20042^{\text {nd }}$ Int. IEEE Conf. "Intelligent Systems", Vol.1, pp.353-358, Bulgaria.

Tsankova, D.D. and V.S. Georgieva (2004b). Stigmergy Based Foraging Behaviour Control with Enhanced Sensing of Object Concentration. In: Proc. of the Int. Conf. on Automatics and Informatics'04, pp.219-222, Bulgaria.

Tsankova, D.D. and A.V. Topalov (1999). Behaviour Arbitration for Autonomous Mobile Robots Using Immune Networks. In: Proc. of the $1^{\text {st }}$ IFAC Workshop on MAS'99, pp.25-30, Austria. 